Note: This is an uncorrected version of an author's manuscript accepted for publication.

*Copyediting, typesetting, and review of the resulting proofs will be undertaken on this manuscript before final publication. During production and prepress, errors may be discovered that could affect the content.

\title{
The relationship between cortisol and cognitive function in healthy older people: the moderating role of ApolipoproteinE polymorphism
}

Teresa Montoliu ${ }^{*}{ }^{1}$, Vanesa Hidalgo ${ }^{1,2,3}$, Matias M. Pulopulos ${ }^{1,4}$, José Luis Ivorra ${ }^{5}$, María José Martínez ${ }^{5}$, and Alicia Salvador ${ }^{1}$

${ }^{1}$ Laboratory of Social Cognitive Neuroscience, IDOCAL, University of Valencia, Valencia (Spain)

${ }^{2}$ Department of Psychology and Sociology, Area of Psychobiology, University of Zaragoza, Teruel, Zaragoza (Spain)

${ }^{3}$ Aragon Health Research Institute, Zaragoza (Spain)

${ }^{4}$ Department of Experimental Clinical and Health Psychology, Ghent University, Ghent (Belgium)

${ }^{5}$ Department of Genetics, University of Valencia, Burjassot, Valencia (Spain)

*Corresponding author: Teresa Montoliu

Laboratory of Social Cognitive Neuroscience, IDOCAL, Faculty of Psychology, University of Valencia, Blasco Ibáñez, 21, 46010, Valencia, Spain.

E-mail address: Teresa.Montoliu@uv.es 


\begin{abstract}
The Apolipoprotein E4 (ApoE4) allele has been suggested as the main risk factor for late onset Alzheimer's disease (AD), whereas the ApoE2 allele has been proposed as a protective factor. These proposals have increased the interest in the effect of the ApoE genotype in healthy people. Additionally, high cortisol levels have been related to negative effects on cognition. The aim of this study was to evaluate cognition (declarative and working memory, attention, and executive function) and its relationship with cortisol, considering the ApoE2, ApoE3, and ApoE4 alleles in healthy older people (55-77 years old). Two saliva samples were collected during the neuropsychological session to obtain cortisol levels and the ApoE genotype. Results showed an association between the ApoE genotype and declarative memory, specifically learning ability, where ApoE2 performed better than ApoE4 and ApoE3. No differences in cortisol levels were obtained considering the ApoE genotype. In addition, higher cortisol levels were related to worse performance on declarative memory. Taking into account the three allelic variations, this association was only significant for the ApoE4 group. On the other hand, higher cortisol levels were related to better attention in all participants. Considering the ApoE groups, this association was only significant for the ApoE3 group. Therefore, our results suggest that carrying the ApoE4 allele may be a vulnerability factor to the adverse effects of Hypothalamic-Pituitary-Adrenal (HPA axis) dysregulation on cognition during aging.
\end{abstract}

Keywords: Apolipoprotein E, Cortisol, Cognition, Memory, Older people 


\section{Introduction}

The increase in life expectancy in our society has aroused interest in the biological mechanisms underlying cognition and its decline during aging (Payton et al., 2005). The Apolipoprotein E (ApoE) polymorphism has been proposed as a mediator of this age-related cognitive impairment (Helkala et al., 1996).

The ApoE gene has three common allelic variations (Bertram, Lill, \&Tanzi, 2010), with the ApoE 3 being the most common, with an occurrence rate of approximately $78 \%$. By contrast, The ApoE2 and ApoE4 alleles are less common (8\% and 14\%, respectively) (Menzel, Kladetzky, \&Assmann, 1983). The ApoE4 allele presents the main risk factor for late-onset $\mathrm{AD}$ (Bertram et al., 2010), and it has also been associated with a higher risk of Mild Cognitive Impairment (MCI) (Dixon et al., 2014), accelerated cognitive decline in normal aging (Bretsky, Guralnik, Launer, Albert, \&Seeman, 2003; Caselli et al., 2004; 2011; Schiepers et al., 2012; Smith, 2002), and decreased longevity (Corder et al., 1996). By contrast, the ApoE2 allele has been associated with survival in older people (Corder et al., 1996) and a reduced risk of AD (Verghese, Castellano, \& Holtzman, 2011). In addition, Farrer et al. (1997) observed that the association between ApoE4 and a higher risk for $\mathrm{AD}$ was stronger in the Caucasian population, compared to African Americans and Hispanics, whereas the ApoE2/E3 genotype appeared to be equally protective across ethnic groups. However, it is important to note that the ApoE gene has no effect on the risk of dementia and mortality in the oldest old (Corrada, Paganini-Hill, Berlau, \& Kawas, 2013).

All these findings have aroused interest in the role ApoE variants play in the cognitive functioning of healthy people. Several studies have found an association between the ApoE4 allele and worse cognitive performance (Bondi, Salmon, Monsch, Galasko, \& Butters, 1995; Deary et al., 2004; Flory, Manuck, Ferrell, Ryan, \& 
Muldoon, 2000; Honea, Vidoni, Harsha, \& Burns, 2009; Kang, Logroscino, De Vivo, Hunter, \& Grodstein, 2005; Small, Rosnick, Fratiglioni, \& Bäckman, 2004; Wilson, Bienias, Berry-Kravis, Evans, \& Bennett, 2002). However, other studies have found no association (Cohen, Small, Lalonde, Friz, \& Sunderland, 2001; Jorm et al., 2007; Lineweaver, Bondi, Galasko, \& Salmon, 2014; Small et al., 2000; Smith et al., 1998), or even better cognitive performance (Alexander et al., 2007; Mondadori et al., 2007; Riley et al., 2000). These inconsistent results may be due to several factors. First, some studies may have included undetected preclinical cases of AD. Therefore, some of the ApoE4-related deficits may be attributable to an overrepresentation of preclinical cases of $\mathrm{AD}$, rather than reflecting the effect of this allele alone (Bondi, Salmon, Galasko, Thomas, \& Thal, 1999). In addition, due to the association between ApoE and AD, where declarative memory is the most affected cognitive function, most of the research has focused on this association, with less research carried out on working memory, attention, and executive function. Moreover, in most studies, the ApoE2 allele has been grouped within the ApoE3 homozygotes under the broad category of "ApoE4 noncarriers" (Beluche, Carrière, Ritchie, \& Ancelin, 2010; Fiocco, Poirier, Joober, Nair, \& Lupien, 2008). Therefore, few studies have explored the effect of the ApoE4 allele independently from the ApoE2 effects (Lara et al., 2013).

In addition to the effects of the ApoE, cognitive function is also influenced by the Hypothalamic-Pituitary-Adrenal (HPA) axis function thorough the influence of cortisol, the end product of HPA-axis activation, on glucocorticoid receptors located in the hippocampus and other brain areas. Hence, higher cortisol levels also have an influence on human cognition in healthy older people (Almela, van der Meij, Hidalgo, Villada, \& Salvador, 2012; Hidalgo, Almela, Pulopulos, \& Salvador, 2016; Lupien, Gillin, \& Hauger, 1999). Increased cortisol levels are a well-established feature in AD, 
although the mechanism responsible for HPA-axis hyperactivity is unknown (Gil-Bea et al., 2010; Peskind, Wilkinson, Petrie, Schellenberg, \& Raskind, 2001). This fact, along with the evidence that ApoE4 is a risk factor for developing AD (Bertram et al., 2010), suggests that the ApoE gene may affect the association between the HPA-axis and cognitive function (Peavy et al., 2007). Moreover, similar to preclinical brain changes related to $\mathrm{AD}$, structural and functional abnormalities of the hippocampus have been found in healthy ApoE4 carriers (Lu et al., 2011). Furthermore, it is worth noting that some results from studies in animals have found that the interaction between the ApoE4 allele and stress could shape cognitive function (Grootendorst et al, 2002; Kloet et al., 2002). In humans, an association between increased risk of developing PTSD and the ApoE4 (Roby, 2017) and ApoE2 alleles (Kim et al., 2013) has been reported; this latter allele has also been related to an increased susceptibility to stress-induced impairments in memory (Freeman, Roca, Guggenheim, Kimbrell, \& Griffin, 2005) and to HPA axis dysregulation (Johnson et al., 2015).

Studies in healthy people are scarce, with varying results. Some studies found an association for the ApoE4 allele between a HPA-axis alteration and cognitive decline (Gerritsen, Comijs, Deeg, Penninx, \& Geerlings, 2011; Lee et al., 2008; Li et al., 2006) measuring salivary cortisol. However, other studies using salivary (Beluche et al., 2010; Li et al., 2006), serum (Lara et al., 2013; Fiocco et al., 2008) or CFS samples (Gil-Bea et al., 2010) to determine cortisol concentrations found no association in healthy individuals. Therefore, this is an issue that warrants additional research.

With all this in mind, we aimed to examine: (i) the differences between the different ApoE groups in cognitive performance (i.e. declarative and working memory, attention, and executive function) and cortisol levels during the neuropsychological assessment and (ii) the relationship between cortisol and cognitive performance, taking 
into account the ApoE groups, in non-stressed, healthy older people. To do so, a neuropsychological battery was administered to assess a wide range of cognitive functions. Based on previous literature, we hypothesized that the ApoE4 group would show worse cognitive performance (Honea et al., 2009; Laukka et al., 2013; Nilsson et al., 2006; Small et al., 2004; Wisdom, Callahan, \& Hawkins, 2011), whereas the ApoE2 group would show better performance (Verghese et al., 2011). We also hypothesized that there would be higher cortisol levels in the ApoE4 group compared to the ApoE2 and ApoE3 groups. Finally, we expected to find an association between higher cortisol levels and worse cognitive function, especially on declarative memory, and this would be more pronounced in the ApoE4 group.

\section{Methods}

\subsection{Participants}

The final sample was composed of 84 participants of both sexes ( 40 men and 44 woman), ranging in age from 55 to 77 years $(M=65.18, S D=4.631)$. Most of the participants had an educational level beyond high school (79.8\%), and their subjective socioeconomic status was medium $(M=5.51, S D=1.086)$ (subjective SES scale: Adler et al., 2000).

The total sample was categorized in three groups: ApoE2 (n=9; E2/E2=1 and $\mathrm{E} 2 / \mathrm{E} 3=8), \operatorname{ApoE} 3(\mathrm{E} 3 / \mathrm{E} 3=59)$, and ApoE4 $(\mathrm{n}=16 ; \mathrm{E} 4 / \mathrm{E} 4=2$ and $\mathrm{E} 4 / \mathrm{E} 3=14)$. Due to the small number of participants who were homozygous for the E2 and E4 allele (E2/E2=1 and $\mathrm{E} 4 / \mathrm{E} 4=2$ ), both the homozygous and heterozygous participants were grouped in the broad category of ApoE2 and ApoE4, respectively. The ApoE group frequencies were $10.7 \%$ for ApoE2, $70.2 \%$ for ApoE3, and $19 \%$ for ApoE4. There were no significant 
differences among the ApoE2, ApoE3, and ApoE4 groups on sex, age, Body Mass Index (BMI), SES, or educational level (all $p>0.115$ ) (Table 1).

\begin{tabular}{lccccccccc}
\hline & $\begin{array}{c}\mathbf{N} \\
(\mathbf{\%})\end{array}$ & $\begin{array}{c}\text { Women } \\
(\%)\end{array}$ & $\begin{array}{c}\text { Age } \\
(\boldsymbol{M})\end{array}$ & $(\boldsymbol{S D})$ & $\begin{array}{c}\text { SES } \\
(\boldsymbol{M})\end{array}$ & $(\boldsymbol{S D})$ & $\begin{array}{c}\text { BMI } \\
(\boldsymbol{M})\end{array}$ & $(\boldsymbol{S D})$ & $\begin{array}{c}\text { Educational } \\
\text { level }\end{array}$ \\
\hline Total & $\begin{array}{c}84 \\
(100 \%)\end{array}$ & $52.4 \%$ & 65.18 & 4.631 & 5.51 & 1.086 & 27.34 & 3.248 & $79.8 \%$ \\
ApoE2 & $\begin{array}{c}9 \\
(10.7 \%)\end{array}$ & $55.6 \%$ & 62.78 & 3.383 & 5.67 & .707 & 27.81 & 2.693 & $77.8 \%$ \\
ApoE3 & $\begin{array}{c}59 \\
(70.2 \%)\end{array}$ & $49.2 \%$ & 65.27 & 4.634 & 5.62 & 1.040 & 27.35 & 3.405 & $81.4 \%$ \\
ApoE4 & 16 & $62.5 \%$ & 66.19 & 4.996 & 5.00 & 1.317 & 27.09 & 3.050 & $75 \%$ \\
& $(19 \%)$ & & & & & & & & \\
& & $p=.625$ & $p=.203$ & & $p=.115$ & & $p=.881$ & & $p=.627$ \\
\hline
\end{tabular}

Table 1. Descriptive data for the total sample and for each ApoE group (ApoE2, ApoE3, and ApoE4). Age, SES (subjective socioeconomic status), and BMI (body mass index) are represented as mean values $(M)$ and standard deviation $(S D)$. Educational level is represented as the percentage of participants with an educational level beyond high school (\%). Differences between ApoE status were analyzed with ANOVAs (age, SES, and BMI) and chi-square (sex and educational level) analyses. No differences in sex, age, SES, BMI, or educational level were found depending on the ApoE group (all $p \geq .115)$.

All the women were postmenopausal and had their last menstrual period more than 3 years before the testing time. None of the participants scored less than 28 on the MEC (Spanish version of the Mini-Mental Status Examination; Lobo et al., 1999), indicating the absence of cognitive impairment.

Participants were Caucasians, and they were recruited from a study program at the University of Valencia for people over 55 years of age. Two hundred and twenty volunteers were interviewed by telephone in order to check whether they met the study prerequisites. Exclusion criteria were: smoking more than 10 cigarettes a day, alcohol or other drug abuse, visual or hearing problems, diabetes, neurological or psychiatric disease, using any medication directly related to emotional or cognitive functioning or able to influence hormonal levels such as glucocorticoids, psychotropic substances, or sleep medications, having been under general anesthesia once or more than once in the past year, and the presence of a stressful life event during the past year. 
One hundred twenty-eight individuals participated in the study, of whom thirty-seven were eliminated because they did not meet the inclusion or exclusion criteria: 20 participants for using medication related to emotional or cognitive functioning or able to influence hormonal levels, such as glucocorticoids, psychotropic substances, or sleep medications; 7 participants for having diabetes; 4 participants due to severe and uncorrected visual or hearing problems; 4 participants for having a stressful life event during the past year; 1 for alcohol abuse; and 1 participant for having been under general anesthesia in the past year. Of the remaining ninety-one participants, the ApoE polymorphism of three participants could not be genotyped, and one participant was excluded for being ApoE2/E4. Finally, after dividing the sample according to the ApoE genotype (ApoE2, ApoE3 and ApoE4), three participants in the ApoE3 group were excluded from the analyses, two participants because their cortisol concentrations differed by more than 2.5 SD from the mean, and one participant because his age differed by more than $2.5 \mathrm{SD}$ from the mean.

\subsection{Procedure}

Participants who met the criteria were asked to attend one session that took place from 10:00 to 12:00 hours in a laboratory at the Faculty of Psychology. Before the session, participants were asked to maintain their general habits, sleep as long as usual, refrain from heavy physical activity the day before the session, and not consume alcohol from the night before the first session. They were also instructed to drink only water, and not eat, smoke, take any stimulants (such as coffee, cola, caffeine, tea or chocolate), or brush their teeth at least 1 hour prior to the session. All participants provided written informed consent to participate in the study, which was conducted in accordance with the Declaration of Helsinki. The protocol was approved by the Research Ethics Committee of the University of Valencia. In this session, a neuropsychological battery 
was administered. Additionally, participants were asked to provide two saliva samples (pre and post neuropsychological assessment), from which the ApoE genotype and cortisol levels were extracted.

\subsection{Neuropsychological tests}

\subsubsection{Declarative memory.}

2.3.1.1. Rey Auditory Verbal Learning Test (RAVLT). The Spanish version of the RAVLT (Miranda \& Valencia, 1997) was used. This task consists of a target list (List A) of 15 neutral words repeated five times by the experimenter (trials I-V: Total Learning) that participants had to learn. Then, an interference list (List B) was presented only once, and participants had to repeat it. Participants were asked to recall the target list again immediately after the interference list (trial VI), and again after a delay of 20 min (trial VII). Three outcomes were used in subsequent analyses: (i) RAVLT Total Learning: total number of words recalled on the first five trials (trial I to V); (ii) RAVLT Immediate Recall: percentage of total number of words recalled after the interference trial compared to the number of words recalled on trial $\mathrm{V}$ (trial $\mathrm{VI} /$ trial $\mathrm{V} \mathrm{x}$ 100); and (iii) RAVLT Delayed Recall: percentage of total number of words recalled after the 20-min delay compared to the number of words recalled on the immediate recall trial (trial VII/trial VI x 100).

2.3.1.2. Rivermead Stories Subtest. The Story recall subtest from the Spanish version of the Rivermead Behavioral Memory Test (Wilson, Cockburn, \&Baddeley, 1985) was used. The experimenter read aloud two short stories, and participants had to recall as many memory units or "ideas" as possible immediately after their oral presentation and after a 20-min delay. Participants' answers were audio recorded and corrected by an expert, and the sum of the correctly recalled "ideas" from the two 
stories was calculated. From this test, two outcomes were used for the subsequent analysis: (i) Rivermead Immediate recall: total "ideas" recalled from the two stories immediately after the oral presentation and (ii) Rivermead Delayed recall: total "ideas" recalled from the two stories after $20 \mathrm{~min}$, compared to the number of "ideas" recalled from the two stories immediately after the oral presentation (Delayed recall/ Immediate recall $\mathrm{x} 100)$.

\subsubsection{Working memory (WM).}

2.3.2.1. Digit Span (DS). The Spanish version of the Wechsler Memory Scale III was administrated (Wechsler, 1997). The experimenter read aloud a series of numbers (from 0 to 9) of increasing length (from 2 to 9 digits) at a rate of one digit per second. The participant had to repeat the numbers, first in the same order (DS-Forward) and then in reverse order (DS-Backward). Each set length was tested twice. The test was finalized when the participant failed two consecutive trials of the same length. For each correctly repeated digit set, one point was given. Two outcomes were obtained: (i) DSForward: total number of correctly recalled attempts in the same order and (ii) DSBackward: total number of correctly recalled attempts in the reverse order. DS-Forward was used as a measure of the attention and memory span component of WM, whereas DS-Backward was used as a measure of the executive component of WM (Conklin, Curtis, Katsanis, Iacono, 2000).

2.3.2.2.Letter-number sequencing (LNS). The Spanish version of the Wechsler Memory Scale III was administrated (Wechsler, 1997). The experimenter read aloud a series of mixed numbers (from 0 to 9 ) and letters (from A to Z) of increasing length (from 2 to 8 items). The participant had to repeat the series, ordering the numbers in ascending order and the letters in alphabetical order. Each set length was tested three times. The test was finalized when the participant failed three consecutive trials of the 
same length. One point was given for each correctly recalled attempt. One outcome was obtained: LNS (total number of correctly recalled attempts).

\subsubsection{Executive Function.}

2.3.3.1.Trial-Making Test (TMT). The TMT (Reitan, 1992) consists of two trials, TMT-A and TMT-B, each composed of 25 circles distributed on a white sheet of paper. In TMT-A, the circles were numbered from 1 to 25 , and the participant was asked to trace a line connecting the circles in numerical sequence as quickly as possible. TMT-B included numbers from 1 to 13 and letters from A to L, and the participant was instructed to alternate between numbers and letters in ascending sequence. The score obtained was the number of seconds required to finish each trial. Errors were pointed out instantly by the examiner and contributed to the score due to the additional time needed for corrections. Two outcomes were obtained: (i) TMT-A: total number of seconds required to finish the TMT-A, and (ii) TMT-B: total number of seconds required to finish the TMT-B. The TMT-A was used to assess attention and general psychomotor speed, whereas the TMT-B was used to evaluate attention-switching performance.

2.3.3.2 .Stroop Color-Word Interference test. Golden's version of the Stroop Color-Word Interference Test(Golden, 1978) was administered. The test is composed of three trials. In each trial, participants had to name as many words as possible in 45 seconds. In the first trial, participants had to read the written word (W), which was red, blue, or green. In the second trial, participants had to name the printed color $(\mathrm{C})$, red, blue, or green, of the XXX letters. In the third trial, participants had to name the color of the printed word (red, blue, or green), which was different from the written word (red, blue, or green) (WC), for example, the word green printed in red color. Afterwards, the $\mathrm{WC}^{\prime}$ was calculated $\left(\mathrm{WC}^{\prime}=(\mathrm{W} \times \mathrm{C}) /(\mathrm{W}+\mathrm{C})\right)$. Finally, the Stroop Interference 
outcome was obtained (Stroop Interference $=\mathrm{WC}-\mathrm{WC}$ '), which is a measure of the ability to inhibit automatic responses.

\subsection{Biochemical analyses}

Participants provided two saliva samples by using salivettes (Sarstedt, Nümbrecht, Germany), the first one at the beginning of the neuropsychological assessment, and the second one at the end of the session. Participants were instructed to keep the cotton swab in their mouths for exactly 2 min, not chew the cotton, and move the swab around in a circular pattern to collect saliva from all salivary glands. The samples were centrifuged at $3000 \mathrm{rpm}$ for $5 \mathrm{~min}$, resulting in a clear supernatant of low viscosity that was stored at $-80^{\circ} \mathrm{C}$ until the analyses of cortisol and ApoE genotype determination were performed.

\subsubsection{Salivary Cortisol.}

The activity of the HPA-axis was measured by analyzing the salivary cortisol levels. Each sample was measured in duplicate, and each participant's samples were analyzed in the same trial. The salivary cortisol samples were analyzed by a competitive solid phase radioimmunoassay (tube coated), using the commercial kit Spectria Cortisol RIA (cat. Nu 06119) from Orion Diagnostica (Espoo, Finland). Assay sensitivity was $0.8 \mathrm{nmol} / \mathrm{L}$, and the within- and inter-assay variation coefficients were all below $8 \%$. Salivary cortisol levels were determined in the Central Research Unit of the Faculty of Medicine, University of Valencia (Spain).

\subsubsection{ApoE genotype determination.}

To determine the ApoE genotype, the genomic deoxyribonucleic acid (DNA) was isolated from the saliva by using a standard commercial extraction method (KIT REALPURE “SSS”). The genotype of each ApoEpolymorphism was amplified by the 
polymerase chain reaction (PCR) using two primers: Forward

(ACAGAATTCGCCCCGGCCTGGTACAC) and Reverse

(TAAGCTTGGCACGCCTGTCCAAGGA). Subsequently, DNA was digested with the Hhal restriction enzyme. Next, DNA electrophoresis in 2\% agarose gel was performed. Finally, after ethidium bromide staining, the DNA-banding patterns (ApoE2, ApoE3 and ApoE4) were visualized under the ultraviolet lamp and recorded for further analysis. The ApoE genotype was determined at the Department of Genetics of the University of Valencia (Spain).

\subsection{Statistical Analysis}

Participants' characteristics were described using percentages or means (standard deviation, SD) when appropriate, according to the ApoE groups (ApoE2, ApoE3, and ApoE4).

To investigate whether there were differences between the ApoE groups in age, BMI, and SES, one-way analyses of variance (ANOVAs) were performed. Pearson's Chi-square test was used to assess differences in sex and education level.

The mean cortisol index was obtained from the cortisol levels presented at baseline (pre-test cortisol) and at the end of the session (post-test cortisol). Before statistical analyses were performed, cortisol data were checked for normal distribution and homogeneity of variance using Shapiro-Wilks and Levene's test. These analyses revealed significant deviations in cortisol values; therefore, cortisol data were logarithm $10(\log 10)$ transformed.

To investigate whether there were differences in cognitive performance and mean cortisol levels among the ApoE groups, we performed one-way ANOVAs. As dependent variables, we used the following outcomes from the (i) RAVLT: RAVLT Total Learning, RAVLT Immediate recall, and RAVLT Delayed recall, (ii) Rivermead 
stories: Rivermead Immediate recall and Rivermead Delayed recall, (iii) DS: DSForward and DS-Backward, (iv) LNS, (v) TMT: TMT-A and TMT-B, (vi) Stroop interference, and (vii) $\log 10$ mean cortisol. Post-hoc comparisons were performed using Bonferroni adjustments for the $p$ values.

To determine whether an association exists between mean cortisol levels and the different cognitive domain outcomes, first, Pearson correlational analyses were conducted. Then, linear regressions were performed in order to investigate these associations, controlling for possible confounder effects. Thus, in separate analyses for each cognitive domain as dependent variable, sex, age, BMI, SES, and educational level were included in step one as covariates, following by stepwise analysis. In step two, we included $\log 10$ mean cortisol. Finally, in order to investigate whether the relationships between the mean cortisol levels and the different cognitive domain outcomes were different depending on the ApoE group, linear regression analyses were repeated with the sample divided into ApoE groups.

All $p$ values were two-tailed, and the level of significance was taken as $p<0.05$. To perform the statistical analyses, version 22.0 of SPSS was used.

\section{Results}

\subsection{ApoE group differences in cognitive function}

One-way ANOVAs revealed significant effects of the ApoE groups on Total Learning from the RAVLT test $(\mathrm{F}(2,81)=4.191, p=.019)$. Post hoc analysis revealed significant differences between the ApoE2 and ApoE4 groups $(p=.015)$, showing that the ApoE2 group obtained higher scores than the ApoE4 group. A marginal difference was also observed, where the ApoE2 group obtained higher scores than the ApoE3 group $(p=.065)$ (Figure 1). None of the other cognitive domain outcomes showed 
significant differences among the ApoE groups. For the rest of the tests that evaluated declarative memory, no significant differences were found between the ApoE groups on immediate $(\mathrm{F}(2,81)=1.298, p=.279)$ and delayed recall $(\mathrm{F}(2,81)=2.552, p=.084)$ from the RAVLT test, or immediate $(\mathrm{F}(2,80)=.034, p=.967)$ and delayed recall $(\mathrm{F}$ $(2,80)=.791, p=.457)$ from the Rivermead test. Likewise, no significant differences were found between the ApoE groups on the tests that evaluated working memory, such as the DS-Forward $(\mathrm{F}(2,81)=1.113, p=.333)$, DS-Backward $(\mathrm{F}(2,80)=.228, p=$ $.796)$, and LNS $(\mathrm{F}(2,81)=.694, p=.503)$. Finally, no significant differences were found between the ApoE groups on the tests that assessed executive function attention, such as TMT-A $(\mathrm{F}(2,80)=1.212, p=.303)$, TMT-B $(\mathrm{F}(2,80)=.329, p=.720)$, and Stroop interference $(\mathrm{F}(2,78)=.360, p=.699)$.

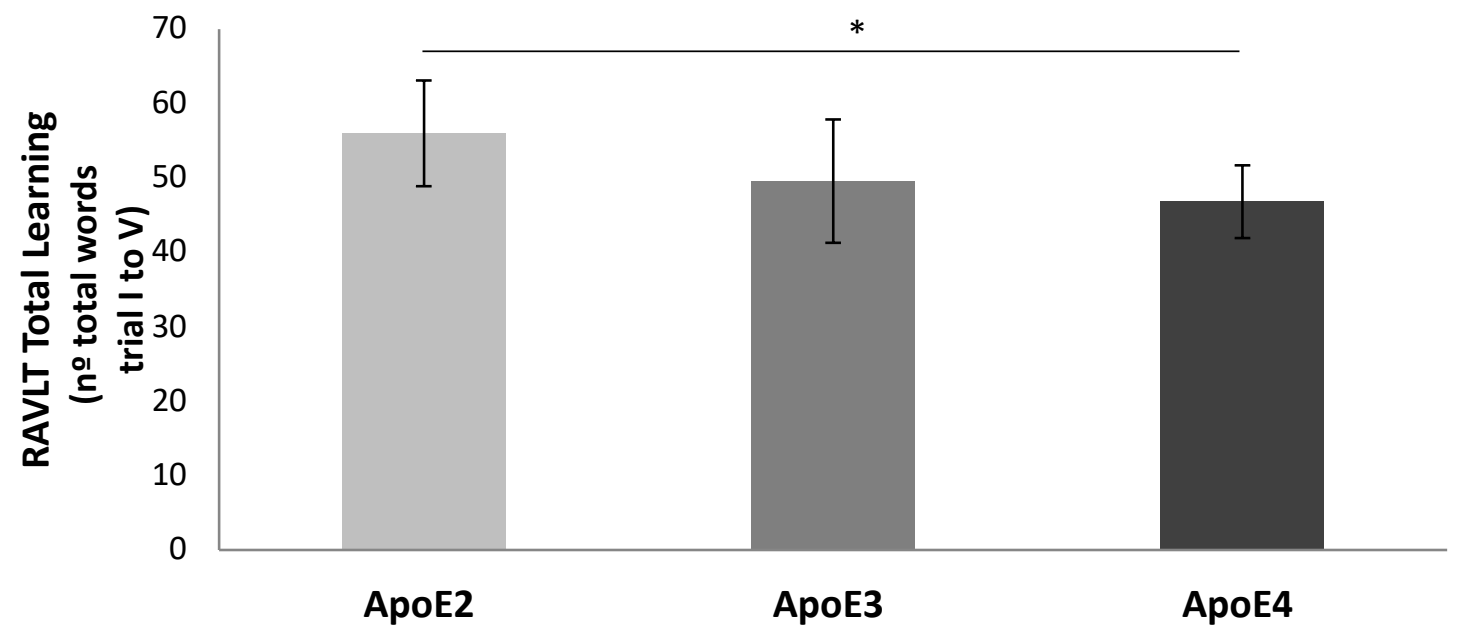

Figure 1. Mean performance on RAVLT Total Learning according to APOE group. One-way ANOVAs showed significant differences in declarative memory performance between ApoE groups ( $p=.019)$. Post hoc analysis showed better performance in the ApoE2 group compared to ApoE4 $(* p=.015)$. Although without reaching significance, a trend was also observed where the ApoE2 group performed better than the ApoE3 group $(p=.065)$.

\subsection{ApoE group differences in mean cortisol levels}


The results showed no significant differences in mean cortisol levels among the ApoE groups $(\mathrm{F}(2,80)=1.351, p=.265)($ Table 2$)$.

\begin{tabular}{ccccccccc}
\hline & \multicolumn{2}{c}{ Total } & \multicolumn{2}{c}{ ApoE2 } & \multicolumn{2}{c}{ ApoE3 } & \multicolumn{2}{c}{ ApoE4 } \\
\hline & $M$ & $S E M$ & $M$ & $S E M$ & $M$ & $S E M$ & $M$ & $S E M$ \\
Mean & 5.7471 & .28650 & 6.4377 & .65110 & 5.7339 & .31741 & 5.4065 & .88720 \\
$\begin{array}{c}\text { Cortisol } \\
(\text { nmol/L) }\end{array}$ & & & & & & & & \\
\hline
\end{tabular}

Table 2. Cortisol data for the total sample and for each ApoE group (ApoE2, ApoE3, and ApoE4) are represented as mean values $(M)$ and standard error of mean $(S E M)$.

\subsection{Relationship between mean cortisol levels and cognitive function}

Pearson correlational analyses showed, for the whole sample, a negative correlation between mean cortisol levels during the neuropsychological session and the results for delayed recall on the RAVLT test $(\mathrm{r}=-.284, p=.009)$ and marginally with immediate recall on the Rivermead story test $(\mathrm{r}=-.196, p=.077)$. After repeating these analyses considering the ApoE groups, only the ApoE4 group showed a negative correlation between cortisol levels and delayed recall on the RAVLT ( $\mathrm{r}=-.570, p=$ $.021)$, and with immediate recall on the Rivermead story test $(\mathrm{r}=-.609, p=.012)$. In turn, for the whole sample, a negative correlation was observed between cortisol levels and time performing the TMT A $(\mathrm{r}=-.230, p=.037)$. After repeating these analyses considering the ApoE groups, it was observed a negative correlation in the ApoE3 group $(\mathrm{r}=-.309, p=.019)($ Table 3$)$.

[Insert Table 3 about here]

Regression analyses showed that, for the complete sample, higher mean cortisol levels during the neuropsychological session were related to worse performance on delayed recall on the RAVLT test $(p=.009)$ and immediate recall on the Rivermead story test $(p=.026)$. After repeating these analyses considering the ApoE groups, this relationship was significant for the ApoE4 group ( $p=.015$ and $p=.008$, respectively). 
In turn, for the whole sample, a trend was observed where higher cortisol levels were associated with lower times performing the TMT A $(p=.060)$ and, therefore, better performance. After repeating these analyses considering the ApoE groups, this relationship was significant for the ApoE3 group $(p=.024)$. None of the other associations were significant (all $p>$.098) (Table 4).

[Insert Table 4 about here]

\section{Discussion}

The aims of the present study were to examine (i) the differences between the ApoE groups in cognitive performance (i.e. declarative and working memory, attention, and executive function) and cortisol levels during the neuropsychological assessment and (ii) the association between cortisol levels and cognitive performance in healthy older people, taking into account the ApoE group (i.e. ApoE2, ApoE3, and ApoE4). To do so, we explored these possible relationships, firstly in the complete sample and then for each ApoE group. The results showed significant differences among the ApoE groups only on declarative memory, specifically on learning ability (RAVLT Total Learning). Moreover, for the complete sample, higher cortisol levels were related to worse performance on declarative memory (delayed recall on the RAVLT and immediate recall on the Rivermead tests). After performing these analyses taking into account the ApoE group, this association was only significant for the ApoE4 group. On the other hand, for the whole sample, higher cortisol levels were marginally related to better attention (i.e. lower times performing the TMT-A). After splitting the sample considering the ApoE groups, this relationship was significant only for the ApoE3 group. 
Our findings demonstrated an association between declarative memory, specifically learning ability, and the ApoE genotype, where subjects carrying the ApoE2 allele performed better than those with ApoE4 and ApoE3. Other studies with older people have also found similar results (Deary et al., 2004; Helkala et al., 1995; 1996; Kang et al., 2005; Wilson et al., 2002). Helkala et al. (1995; 1996) also observed that the ApoE2 group was associated with better learning ability than the ApoE3 and ApoE4 groups. Moreover, in a review, Wisdom et al. (2011) reported that the ApoE2 group performed better on declarative memory than theApoE4 group. Thus, our results, coinciding with other studies, support the idea that the ApoE2 allele provides an advantage on declarative memory performance, compared to the ApoE3 and ApoE4 alleles.

No differences were found between the ApoE3 and ApoE4 groups on declarative memory performance. This finding was surprising because we expected worse performance on declarative memory in the ApoE4 group, as in previous studies (Honea et al., 2009; Laukka et al., 2013; Nilsson et al., 2006; Small et al., 2004; Wisdom et al., 2011). However, some studies have reported that cognitive deficits related to the ApoE4 allele were due to preclinical cases of $\mathrm{AD}$, and that this association disappeared when possible dementia cases were removed (Batterham, Bunce, Cherbuin, \& Christensen, 2013;Salo et al., 2001). It is important to note that, in our study, all participants scored 28 on the MEC, indicating no cognitive impairment. In addition, participants underwent very restrictive criteria compared to other studies, and so a very healthy sample was obtained. Therefore, in our sample of non-demented and non-stressed healthy elderly people, there would be no differences between the ApoE3 and ApoE4 groups. Moreover, in our study, due to the small number of ApoE4 homozygous (E4/E4=2) and heterozygous $(E 3 / E 4=14)$ participants, they were grouped into the broad category of the 
ApoE4 group, leading to an overrepresentation of the ApoE4 heterozygous group. In a meta-analysis, Wisdom et al. (2011) found that people with the ApoE4 homozygote performed significantly worse than ApoE4 non-carriers on declarative memory. However, no significant differences were found between ApoE4 heterozygote and ApoE4 non-carriers. Thus, our findings are consistent with this meta-analysis, where no significant differences where observed between the ApoE4 and the ApoE3 groups on declarative memory tasks. Finally, few studies have explored the effect of the ApoE4 allele taking into account the ApoE2 allele effect. In most studies, the ApoE2 allele, associated with better performance, has been grouped with the ApoE3 allele under the broad category of "ApoE4 non-carriers". Therefore, this may have led to an overestimation of the ApoE3 and an underestimation of the ApoE4 allele performance. Thus, these considerations would reinforce our results showing no differences in cognitive function between the ApoE3 and the ApoE4 groups.

On the other hand, unexpectedly, no differences in cortisol levels were found among the ApoE groups. Some studies have suggested that the relationship between the ApoE4 allele and higher cortisol levels might appear only in AD and dementia (Fiocco et al., 2008; Gil-Bea et al., 2010), which would explain that this association was not observed in our cognitively healthy sample.

Regarding the second aim of the present study, as expected, higher cortisol levels were associated with worse declarative memory performance, both immediate and delayed recall, in the complete sample (Lee et al., 2007; Li et al., 2006; MacLullichet al., 2005; Seeman, McEwen, Singer, Albert, \& Rowe, 1997;Vedhara, Hyde, Gilchrist, Tytherleigh, \& Plummer, 2000; Wright, Kunz-Ebrecht, Iliffe, Foese, \& Steptoe, 2005) and in the ApoE4 group (Gerretsein et al., Singh-Manoux et al., 2014). Previous studies found an association between the ApoE4 allele, a faster decline in 
verbal fluency, and a flatter diurnal slope, that is, higher evening and lower morning cortisol (Gerritsen et al., 2011; Singh-Manoux et al., 2014). In turn, as in our study, Lee et al. (2008) measured cortisol levels during the cognitive assessment and observed that, whereas higher cortisol was associated with lower cognitive performance, the slopes were steeper in the ApoE4 group. Thus, in line with our results, they suggested that the ApoE4 allele increases the aging brain's vulnerability to adverse effects of cortisol.

Finally, higher cortisol levels were associated with lower time performing the TMT-A and, therefore, better attention, in the total sample and in the ApoE3 group. Glucocorticoid receptors are widely distributed in the hippocampus and in the prefrontal cortex, which are involved in declarative memory and attention and executive function, respectively (Lupien et al., 1999). Whereas some studies found that lower cortisol levels were related to worse attentional performance (Pulopulos et al., 2015; Vedhara et al., 2000), other studies reported an association between higher cortisol levels and worse attention and processing speed (Beluche et al., 2010; Fonda, Bertrand, O'Donnell, Longcope, \&McKinlay, 2005; Franz et al., 2011;Lee et al., 2007; MacLullich et al., 2005). In addition, Lupien et al. (1999) observed an inverted-U pattern between cortisol and working memory performance that has an attentional component (Mirsky, Anthony, Duncan, Ahearn, \& Kellam, 1991) and is also mediated by prefrontal cortex functioning. They found that, whereas low doses of increased corticosteroids would improve performance, high doses would impair it. Therefore, in our study with elderly people, higher cortisol levels (Lupien, Maheu, Tu, Fiocco, \&Schramek, 2007) may have improved attentional performance. To our knowledge, no studies have found this association in the ApoE3 group. By contrast, Gerritsen et al. (2011) and Lee et al. (2008) observed that the ApoE4 group was more vulnerable to the detrimental effects of cortisol on cognitive functioning. Therefore, the positive relationship between cortisol 
and attention in the ApoE3 group may indicate that they are less vulnerable to the negative effects of corticosteroids on attention.

Some limitations should be considered. First, it is important to note the correlational nature of the results, and so we cannot claim causal relationships. In addition, due to the unequal distribution of the three ApoE alleles and the low frequency of the ApoE2 and ApoE4 alleles in the population, in our sample the number of participants in each ApoE group differed, and the sample size for the ApoE2 and the ApoE4 groups was small. Therefore, it is possible that due to the small sample sizes some of the expected results that had been hypothesized have not been observed. Thus, a larger sample size would be necessary to increase the statistical power. Nevertheless, it is important to highlight the effort that has been made in this study to separate and compare the three allelic variations and study their relationship with a wide range of cognitive domains and the HPA axis. In addition, the strict exclusion criteria make it possible to control the effect of confounding variables. Finally, also due to the small sample size, sex differences have not been taken into account, although several studies with healthy elderly people have shown that women outperform men on verbal memory (Zhang, Zhou, Wang, Zhang \& Study, 2017; Munro et al., 2012), whereas men perform better on visuospatial ability (Munro et al.,2012). Moreover, there is evidence that women are at higher risk for AD than men (Farrer et al, 1997), and so it seems necessary to study sex differences in the influence of the ApoE gene on cognitive function. Whereas some studies have reported a higher ApoE4-associated risk of AD in women than in men (Corrada et al., 2013; Farrer et al., 1997), others found the opposite relationship (Qiu, Kivipelto, Agüero-Torres, Winblad, \& Fratiglioni, 2004) or no association (Yip, Brayne, Easton, \& Rubinsztein, 2002). Thus, future studies with larger samples are needed to shed light on this pending issue. 
In summary, our results show that, in healthy older people, the ApoE2 allele may have a protective effect on declarative memory, specifically learning ability. Additionally, although the ApoE4 allele would not have a negative effect on cognitive function compared to ApoE3, high cortisol levels would be especially detrimental to declarative memory in this group, compared to the ApoE2 and ApoE3 groups. Thus, the ApoE4 allele could add greater vulnerability to the adverse effects of HPA axis dysregulation on declarative memory during aging.

\section{Acknowledgements}

This research study was supported by the Spanish Education and Science Ministry (grant no. PSI2013-46889), by the Generalitat Valenciana (grants no. PROMETEOII2015-020 and ACOMP2015-227), and by the Universitat de València (UV-PREDOC16F1-383576). The authors wish to thank Ms. Cindy DePoy for the revision of the English text.

\section{References}

Alexander, D., Williams, L., Gatt, J., Dobson-Stone, C., Kuan, S., Todd, E., . . G Gordon, E. (2007). The contribution of apolipoprotein $\mathrm{E}$ alleles on cognitive performance and dynamic neural activity over six decades. BiologicalPsychology, 75(3), 229-238. doi:10.1016/j.biopsycho.2007.03.001

Almela, M., Meij, L. V., Hidalgo, V., Villada, C., \& Salvador, A. (2012). The cortisol awakening response and memory performance in older men and women. Psychoneuroendocrinology, 37(12), 19291940.doi:10.1016/j.psyneuen.2012.04.009 
Batterham, P. J., Bunce, D., Cherbuin, N., \& Christensen, H. (2012).Apolipoprotein E $\varepsilon 4$ and Later-Life Decline in Cognitive Function and Grip Strength. American Journal of Geriatric Psychiatry, 1.doi:10.1097/jgp.0b013e318266b1ee

Beluche, I., Carrière, I., Ritchie, K., \&Ancelin, M. L. (2010).A prospective study of diurnal cortisol and cognitive function in community-dwelling elderly people. Psychological Medicine, 40(06), 1039-1049.doi:10.1017/s0033291709991103

Bertram, L., Lill, C. M., \&Tanzi, R. E. (2010). The Genetics of Alzheimer Disease: Back to the Future. Neuron, 68(2), 270-281.doi:10.1016/j.neuron.2010.10.013

Bondi, M. W., Salmon, D. P., Galasko, D., Thomas, R. G., \&Thal, L. J. (1999).Neuropsychological function and apolipoprotein E genotype in the preclinical detection of Alzheimers disease. Psychology and Aging, 14(2), 295303.doi:10.1037//0882-7974.14.2.295

Bondi, M., Salmon, D., Monsch, A., Galasko, D., Butters, N., Klauber, M., . . Saitoh, T. (1995). Episodic memory changes are associated with the APOE- epsilon 4 allele in nondemented older adults. Neurology, 45(12), 2203-2206.doi:10.1212/wnl.45.12.2203

Bretsky, P., Guralnik, J. M., Launer, L., Albert, M., \&Seeman, T. E. (2003). The role of APOE4 in longitudinal cognitive decline: MacArthur Studies of Successful Aging. Neurology, 60(7), 1077-1081.doi:10.1212/01.wnl.0000055875.26908.24

Caselli, R. J., Dueck, A. C., Locke, D. E., Hoffman-Snyder, C. R., Woodruff, B. K., Rapcsak, S. Z., \&Reiman, E. M. (2011). Longitudinal modeling of frontal cognition in APOE 4 homozygotes, heterozygotes, and noncarriers. Neurology, 76(16), 13831388.doi:10.1212/wnl.0b013e3182167147

Caselli, R. J., Reiman, E. M., Osborne, D., Hentz, J. G., Baxter, L. C., Hernandez, J. L., \& Alexander, G. G. (2004). Longitudinal changes in cognition and behavior in 
asymptomatic carriers of the APOE e4 allele. Neurology, 62(11), 19901995.doi:10.1212/01.wnl.0000129533.26544.bf

Cohen, R. M., Small, C., Lalonde, F., Friz, J., \& Sunderland, T. (2001).Effect of apolipoprotein E genotype on hippocampal volume loss in aging healthy women. Neurology, 57(12), 2223-2228.doi:10.1212/wnl.57.12.2223

Conklin, H. M. (2000). Verbal Working Memory Impairment in Schizophrenia Patients and Their First-Degree Relatives: Evidence From the Digit Span Task. American Journal of Psychiatry, 157(2), 275-277.doi:10.1176/appi.ajp.157.2.275

Corder, E. H., Lannfelt, L., Viitanen, M., Corder, L. S., Manton, K. G., Winblad, B., \&Basun, H. (1996).Apolipoprotein E Genotype Determines Survival in the Oldest Old (85 Years or Older) Who Have Good Cognition. Archives of Neurology, 53(5), 418422.doi:10.1001/archneur.1996.00550050048022

Corrada, M. M., Paganini-Hill, A., Berlau, D. J., \& Kawas, C. H. (2013). Apolipoprotein E genotype, dementia, and mortality in the oldest old: the 90+ Study. Alzheimer's \& Dementia, 9(1), 12-18. doi: 10.1016/j.jalz.2011.12.004

de Kloet, E. R., Grootendorst, J., Karssen, A. M., \& Oitzl, M. S. (2002). Gene× environment interaction and cognitive performance: animal studies on the role of corticosterone. Neurobiology of learning and memory, 78(3), 570-577. doi: 10.1006/nlme.2002.4079

Deary, I. J., Whiteman, M. C., Pattie, A., Starr, J. M., Hayward, C., Wright, A. F., . . Whalley, L. J. (2004).Apolipoprotein E Gene Variability and Cognitive Functions at Age 79: A Follow-Up of the Scottish Mental Survey of 1932. Psychology and Aging, 19(2), 367371. doi:10.1037/0882-7974.19.2.367

Dixon, R. A., Decarlo, C. A., Macdonald, S. W., Vergote, D., Jhamandas, J., \&Westaway, D. (2014). APOE and COMT polymorphisms are complementary biomarkers of status, 
stability, and transitions in normal aging and early mild cognitive impairment. Frontiers in Aging Neuroscience, 6.doi:10.3389/fnagi.2014.00236

Farrer, L. A., Cupples, L. A., Haines, J. L., Hyman, B., Kukull, W. A., Mayeux, R., ... \& Van Duijn, C. M. (1997). APOE and Alzheimer Disease Meta Analysis Consortium Effects of age, sex, and ethnicity on the association between apolipoprotein E genotype and Alzheimer disease. A meta-analysis. Jama, 278(16), 1349-1356. doi: 10.1001/jama.278.16.1349

Fiocco, A. J., Poirier, J., Joober, R., Nair, N., \&Lupien, S. J. (2008).Acute and long-term associations between ApoE genetic polymorphism, cortisol levels, and declarative memory performance in older adults. Psychoneuroendocrinology, 33(5), 625633.doi:10.1016/j.psyneuen.2008.02.002

Flory, J. D., Manuck, S. B., Ferrell, R. E., Ryan, C. M., \& Muldoon, M. F. (2000). Memory performance and the apolipoprotein E polymorphism in a community sample of middleaged adults. American Journal of Medical Genetics, 96(6), 707-711.doi:10.1002/10968628(20001204)96:6<707::aid-ajmg1>3.0.co;2-v

Fonda, S. J., Bertrand, R., Odonnell, A., Longcope, C., \&Mckinlay, J. B. (2005). Age, Hormones, and Cognitive Functioning Among Middle-Aged and Elderly Men: CrossSectional Evidence From the Massachusetts Male Aging Study. The Journals of Gerontology Series A: Biological Sciences and Medical Sciences, 60(3), 385-390. doi:10.1093/gerona/60.3.385

Franz, C. E., O’Brien, R. C., Hauger, R. L., Mendoza, S. P., Panizzon, M. S., Prom-Wormley, E., . . Kremen, W. S. (2011). Cross-sectional and 35-year longitudinal assessment of salivary cortisol and cognitive functioning: The Vietnam Era Twin Study of Aging. Psychoneuroendocrinology, 36(7), 10401052.doi:10.1016/j.psyneuen.2011.01.002 
Freeman, T., Roca, V., Guggenheim, F., Kimbrell, T., \& Griffin, W. S. T. (2005).

Neuropsychiatric associations of apolipoprotein E alleles in subjects with combatrelated posttraumatic stress disorder. The Journal of neuropsychiatry and clinical neurosciences, 17(4), 541-543. doi:10.1176/appi.neuropsych.17.4.541

Gerritsen, L., Comijs, H. C., Deeg, D. J., Penninx, B. W., \&Geerlings, M. I. (2011).Salivary cortisol, APOE- $\varepsilon 4$ allele and cognitive decline in a prospective study of older persons. Neurobiology of Aging,32(9), 1615-1625.

doi:10.1016/j.neurobiolaging.2009.09.007

Gil-Bea, F. J., Aisa, B., Solomon, A., Solas, M., Mugueta, M. D., Winblad, B., . . Ramírez, M. J. (2010). HPA Axis Dysregulation Associated to Apolipoprotein E4 Genotype in Alzheimers Disease. Journal of Alzheimers Disease, 22(3), 829-838.doi:10.3233/jad2010-100663

Golden, C.J., 1978.A Manual for the Clinical and Experimental Use of the Stroop Color and Word Test. Stoelting, Wood Dale, IL.

Grootendorst, J., Kempes, M. M., Lucassen, P. J., Dalm, S., De Kloet, E. R., \& Oitzl, M. S. (2002). Differential effect of corticosterone on spatial learning abilities in apolipoprotein E knockout and C57BL/6J mice. Brain research, 953(1), 281-285. doi: $10.1016 / \mathrm{s} 0006-8993(02) 03399-1$

Helkala, E., Koivisto, K., Hänninen, T., Vanhanen, M., Kervinen, K., Kuusisto, J., . . . Riekkinen, P. (1996). Memory functions in human subjects with different apolipoprotein E phenotypes during a 3-year population-based follow-up study. Neuroscience Letters, 204(3), 177-180. doi:10.1016/0304-3940(96)12348-x

Helkala, E., Koivisto, K., Hänninen, T., Vanhanen, M., Kervinen, K., Kuusisto, J., . . .Riekkinen, P. (1995). The association of apolipoprotein E polymorphism with memory: 
a population based study. NeuroscienceLetters, 191(3), 141-144. doi:10.1016/03043940(95)11575-h

Hidalgo, V., Almela, M., Pulopulos, M. M., \& Salvador, A. (2016). Memory performance is related to the cortisol awakening response in older people, but not to the diurnal cortisol slope. Psychoneuroendocrinology, 71, 136-146.doi:10.1016/j.psyneuen.2016.05.019

Honea, R. A., Vidoni, E., Harsha, A., \& Burns, J. M. (2009). Impact of APOE on the Healthy Aging Brain: A Voxel-Based MRI and DTI Study. Journal of Alzheimers Disease, 18(3), 553-564.doi:10.3233/jad-2009-1163

Johnson, L. A., Zuloaga, D. G., Bidiman, E., Marzulla, T., Weber, S., Wahbeh, H., \& Raber, J. (2015). ApoE2 exaggerates PTSD-related behavioral, cognitive, and neuroendocrine alterations. Neuropsychopharmacology, 40(10), 2443-2453. doi: 10.1038/npp.2015.95

Jorm, A. F., Mather, K. A., Butterworth, P., Anstey, K. J., Christensen, H., \&Easteal, S. (2007). APOE genotype and cognitive functioning in a large age-stratified population sample. Neuropsychology, 21(1), 1-8. doi: 10.1037/0894-4105.21.1.1

Kang, J. H., Logroscino, G., Vivo, I. D., Hunter, D., \&Grodstein, F. (2005). Apolipoprotein E, cardiovascular disease and cognitive function in aging women. Neurobiology of Aging, 26(4), 475-484.doi:10.1016/j.neurobiolaging.2004.05.003

Kim, T. Y., Chung, H. G., Shin, H. S., Kim, S. J., Choi, J. H., Chung, M. Y., ... \& Cho, H. S. (2013). Apolipoprotein E gene polymorphism, alcohol use, and their interactions in combat-related posttraumatic stress disorder. Depression and anxiety, 30(12), 11941201. doi: $10.1002 /$ da. 22138

Lara, V. P., Caramelli, P., Teixeira, A. L., Barbosa, M. T., Carmona, K. C., Carvalho, M. G., ...\& Gomes, K. B. (2013). High cortisol levels are associated with cognitive impairment no-dementia (CIND) and dementia. ClinicaChimicaActa, 423, 1822.doi:10.1016/j.cca.2013.04.013 
Laukka, E. J., Lövdén, M., Herlitz, A., Karlsson, S., Ferencz, B., Pantzar, A., ...\&Bäckman, L. (2013). Genetic effects on old-age cognitive functioning: a population-based study. Psychology and aging, 28(1), 262-274.doi:10.1037/a0030829

Lee, B. K., Glass, T. A., Mcatee, M. J., Wand, G. S., Bandeen-Roche, K., Bolla, K. I., \& Schwartz, B. S. (2007). Associations of Salivary Cortisol With Cognitive Function in the Baltimore Memory Study. Archives of General Psychiatry, 64(7), 810818.doi:10.1001/archpsyc.64.7.810

Lee, B. K., Glass, T. A., Wand, G. S., Mcatee, M. J., Bandeen-Roche, K., Bolla, K. I., \& Schwartz, B. S. (2008).Apolipoprotein E Genotype, Cortisol, and Cognitive Function in Community-Dwelling Older Adults. American Journal of Psychiatry, 165(11), 14561464.doi:10.1176/appi.ajp.2008.07091532

Li, G., Cherrier, M. M., Tsuang, D. W., Petrie, E. C., Colasurdo, E. A., Craft, S., . . . Wilkinson, C. W. (2006).Salivary cortisol and memory function in human aging. Neurobiology of Aging, 27(11), 17051714.doi:10.1016/j.neurobiolaging.2005.09.031

Lineweaver, T. T., Bondi, M. W., Galasko, D., \& Salmon, D. P. (2014).Effect of Knowledge of APOE Genotype on Subjective and Objective Memory Performance in Healthy Older Adults. American Journal of Psychiatry, 171(2), 201-208. doi:10.1176/appi.ajp.2013.12121590

Lobo, A., Saz, P., Marcos, G., Día, J. L., de la Cámara, C., Ventura, T., et al. (1999). Revalidación y normalización del mini-examen cognoscitivo (primera versión en castellano del Mini-Mental Status Examination) en la población general geriátrica. Medicina Clínica, 112 (20), 767-774.

Lu, P.H., Thompson, P.M., Leow, A., Lee, G.J., Lee, A., Yanovsky, I., Parikshak, N., Khoo, T., Wu, S., Geschwind, D., Bartzokis, G. (2011). Apoliprotein E genotype is associated 
with temporal and hippocampal atrophy rates in healthy older people adults: a tensorbased morphometry study. Journal of Alzheimer's disease, 23(3),433-42.doi: 10.3233/JAD-2010-101398

Lupien, S. J., Gillin, C. J., \&Hauger, R. L. (1999). Working memory is more sensitive than declarative memory to the acute effects of corticosteroids: A dose-response study in humans. Behavioral neuroscience, 113(3), 420-430.doi: 10.1037//0735-7044.113.3.420

Lupien, S. J., Maheu, F., Tu, M., Fiocco, A., \&Schramek, T. E. (2007).The effects of stress and stress hormones on human cognition: Implications for the field of brain and cognition.Brain and cognition, 65(3), 209-237.doi: 10.1016/j.bandc.2007.02.007

MacLlullich, A. M., Deary, I. J., Starr, J. M., Ferguson, K. J., Wardlaw, J. M., \&Seckl, J. R. (2005).Plasma cortisol levels, brain volumes and cognition in healthy elderly men. Psychoneuroendocrinology, 30(5), 505-515.doi:10.1016/j.psyneuen.2004.12.005

Menzel, H. J., Kladetzky, R. G., \&Assmann, G. (1983).Apolipoprotein E polymorphism and coronary artery disease.Arteriosclerosis, 3(4), 310-315.

Miranda, J. P., \& Valencia, R. R. (1997).English and Spanish versions of a memory test: Wordlength effects versus spoken-duration effects. Hispanic Journal of Behavioral Science, 19(2), 171-181.doi: 10.1177/07399863970192005

Mirsky, A. F., Anthony, B. J., Duncan, C. C., Ahearn, M. B., \&Kellam, S. G. (1991). Analysis of the elements of attention: A neuropsychological approach. Neuropsychology Review, 2(2), 109-145.doi:10.1007/bf01109051

Mondadori, C. R., D. J. - F. De Quervain, Buchmann, A., Mustovic, H., Wollmer, M. A., Schmidt, C. F., .. . Henke, K. (2007). Better Memory and Neural Efficiency in Young ApolipoproteinE 4 Carriers. Cerebral Cortex, 17(8), 1934-1947.

doi:10.1093/cercor/bhl103 
Munro, C. A., Winicki, J. M., Schretlen, D. J., Gower, E. W., Turano, K. A., Muñoz, B., ... \& West, S. K. (2012). Sex differences in cognition in healthy elderly individuals. Aging, Neuropsychology, and Cognition, 19(6), 759-768. doi:10.1080/13825585.2012.690366

Nilsson, L. G., Adolfsson, R., Bäckman, L., Cruts, M., Nyberg, L., Small, B. J., \& Van Broeckoven, C. (2006). The influence of APOE status on episodic and semantic memory: data from a population-based study. Neuropsychology, 20(6), 645-657.doi: $10.1037 / 0894-4105.20 .6 .645$

Payton, A., Gibbons, L., Davidson, Y., Ollier, W., Rabbitt, P., Worthington, J., . . Horan, M. (2005).Influence of serotonin transporter gene polymorphisms on cognitive decline and cognitive abilities in a nondemented elderly population.Molecular Psychiatry, 10(12), 1133-1139.doi:10.1038/sj.mp.4001733

Peavy, G. M., Lange, K. L., Salmon, D. P., Patterson, T. L., Goldman, S., Gamst, A. C., . . .Galasko, D. (2007).The Effects of Prolonged Stress and APOE Genotype on Memory and Cortisol in Older Adults.Biological Psychiatry, 62(5), 472478.doi:10.1016/j.biopsych.2007.03.013

Peskind, E. R., Wilkinson, C. W., Petrie, E. C., Schellenberg, G. D., \&Raskind, M. A. (2001). Increased CSF cortisol in AD is a function of APOE genotype. Neurology, 56(8), 10941098. doi: 10.1212/WNL.56.8.1094

Pulopulos, M. M., Hidalgo, V., Almela, M., Puig-Perez, S., Villada, C., \& Salvador, A. (2015). Acute stress and working memory in older people. Stress, 18(2), 178-187. doi:10.3109/10253890.2015.1004538

Qiu, C., Kivipelto, M., Agüero-Torres, H., Winblad, B., \&Fratiglioni, L. (2004). Risk and protective effects of the APOE gene towards Alzheimer's disease in the Kungsholmen project: variation by age and sex. Journal of Neurology, Neurosurgery \& Psychiatry, 75(6), 828-833. doi:10.1136/jnnp.2003.021493 
Reitan, R.M., 1992. Trail Making Test: Manual for Administration and Scoring. Reitan Neuropsychology Laboratory, (Tucson, AZ).

Riley, K. P., Snowdon, D. A., Saunders, A. M., Roses, A. D., Mortimer, J. A., \&Nanayakkara, N. (2000). Cognitive Function and Apolipoprotein E in Very Old Adults: Findings From the Nun Study. The Journals of Gerontology Series B: Psychological Sciences and Social Sciences, 55(2). doi:10.1093/geronb/55.2.s69

Roby, Y. (2017). Apolipoprotein E variants and genetic susceptibility to combat-related posttraumatic stress disorder: a meta-analysis. Psychiatric Genetics, 27(4), 121-130. doi: 10.1097/ypg.0000000000000174

Salo, A., Ylikoski, R., Verkkoniemi, A., Polvikoski, T., Juva, K., Rastas, S., . . Sulkava, R. (2001). Does Apolipoprotein E Influence Learning and Memory in the NondementedOldest Old? International Psychogeriatrics, 13(4), 451459.doi:10.1017/s1041610201007864

Schiepers, O. J., Harris, S. E., Gow, A. J., Pattie, A., Brett, C. E., Starr, J. M., \&Deary, I. J. (2011). APOE E4 status predicts age-related cognitive decline in the ninth decade: longitudinal follow-up of the Lothian Birth Cohort 1921. Molecular Psychiatry, 17(3), 315-324.doi:10.1038/mp.2010.137

Seeman, T. E., McEwen, B. S., Singer, B. H., Albert, M. S., \& Rowe, J. W. Seeman, T. E. (1997). Increase in Urinary Cortisol Excretion and Memory Declines: MacArthur Studies of Successful Aging. Journal of Clinical Endocrinology \& Metabolism, 82(8), 2458-2465. doi:10.1210/jc.82.8.2458

Singh-Manoux, A., Dugravot, A., Elbaz, A., Shipley, M., Kivimaki, M., \&Kumari, M. (2014). No evidence of a longitudinal association between diurnal cortisol patterns and cognition. Neurobiology of Aging, 35(10), 2239-2245.doi:10.1016/j.neurobiolaging.2014.03.015 
Small, B. J., Graves, A. B., Mcevoy, C. L., Crawford, F. C., Mullan, M., \& Mortimer, J. A. (2000). Is APOE- 4 a risk factor for cognitive impairment in normal aging? Neurology, 54(11), 2082-2088.doi:10.1212/wnl.54.11.2082

Small, B. J., Rosnick, C. B., Fratiglioni, L., \&Bäckman, L. (2004).Apolipoprotein E and Cognitive Performance: A Meta-Analysis. Psychology and Aging, 19(4), 592600.doi:10.1037/0882-7974.19.4.592

Smith, G. E., Bohac, D. L., Waring, S. C., Kokmen, E., Tangalos, E. G., Ivnik, R. J., \& Petersen, R. C. (1998). Apolipoprotein E genotype influences cognitive phenotype in patients with Alzheimers disease but not in healthy control subjects. Neurology, 50(2), 355-362.doi:10.1212/wnl.50.2.355

Smith, J. D. (2002). Apolipoproteins and aging: emerging mechanisms. Ageing Research Reviews, 1(3), 345-365.doi:10.1016/s1568-1637(02)00005-3

Vedhara, K., Hyde, J., Gilchrist, I., Tytherleigh, M., \& Plummer, S. (2000). Acute stress, memory, attention and cortisol. Psychoneuroendocrinology, 25(6), 535549.doi:10.1016/s0306-4530(00)00008-1

Verghese, P. B., Castellano, J. M., \&Holtzman, D. M. (2011).Apolipoprotein E in Alzheimers disease and other neurological disorders. The Lancet Neurology, 10(3), 241252.doi:10.1016/s1474-4422(10)70325-2

Wechsler, D., 1997. WMS-III Administration and Scoring Manual. The Psychological Corporation, Harcourt Brace \& Company, San Antonio.

Wilson, B., Cockburn, J., \&Baddeley, A. (1985).TheRivermeadbehavioural memory test. Reading, UK: Thames Valley Text.

Wilson, R. S., Bienias, J. L., Berry-Kravis, E., Evans, D. A., \& Bennett, D. A. (2002). The apolipoprotein E E2 allele and decline in episodic memory. Journal of Neurology, Neurosurgery \& Psychiatry, 73(6), 672-677.doi: 10.1136/jnnp.73.6.672 
Wisdom, N. M., Callahan, J. L., \& Hawkins, K. A. (2011). The effects of apolipoprotein E on non-impaired cognitive functioning: A meta-analysis. Neurobiology of Aging, 32(1), 6374.doi:10.1016/j.neurobiolaging.2009.02.003

Wright, C., Kunz-Ebrecht, S., Iliffe, S., Foese, O., \& Steptoe, A. (2005).Physiological correlates of cognitive functioning in an elderly population. Psychoneuroendocrinology, 30(9), 826838.doi:10.1016/j.psyneuen.2005.04.001

Yip, A. G., Brayne, C., Easton, D., \&Rubinsztein, D. C. (2002).Apolipoprotein E4 is only a weak predictor of dementia and cognitive decline in the general population. Journal of medical genetics, 39(9), 639-643. doi: 10.1136/jmg.39.9.639

Zhang, J., Zhou, W., Wang, L., Zhang, X., \& Study, H. A. B. (2017). Gender differences of neuropsychological profiles in cognitively normal older people without amyloid pathology. Comprehensive Psychiatry, 75, 22-26.

doi:10.1016/j.comppsych.2017.02.008 


\begin{tabular}{lcccccccccccc}
\hline & & $\begin{array}{c}\text { RAVLT } \\
\text { Total } \\
\text { Learning }\end{array}$ & $\begin{array}{c}\text { RAVLT } \\
\text { Immediate } \\
\text { Recall }\end{array}$ & $\begin{array}{c}\text { RAVLT } \\
\text { Delayed } \\
\text { Recall }\end{array}$ & $\begin{array}{c}\text { Rivermead } \\
\text { Immediate } \\
\text { Recall }\end{array}$ & $\begin{array}{c}\text { Rivermead } \\
\text { Delayed } \\
\text { Recall }\end{array}$ & $\begin{array}{c}\text { DS- } \\
\text { Forward }\end{array}$ & $\begin{array}{c}\text { DS- } \\
\text { Backward }\end{array}$ & $\begin{array}{c}\text { LNS } \\
\text { TMT-A }\end{array}$ & $\begin{array}{c}\text { TMT-B } \\
\text { Stroop } \\
\text { Interfe- } \\
\text { rence }\end{array}$ \\
\hline Total & $\mathrm{r}$ & -.126 & .069 & $\mathbf{- . 2 8 4}$ & -.196 & -.006 & .020 & .006 & -.010 & -.230 & .033 & .024 \\
& $p$ & .256 & .533 & $\mathbf{. 0 0 9}$ & .077 & .956 & .858 & .958 & .928 & $\mathbf{. 0 3 7}$ & .768 & .830 \\
ApoE2 & $\mathrm{r}$ & -.254 & -.020 & .223 & -.518 & -.126 & .233 & .281 & -.454 & -.024 & -.036 & .044 \\
& $p$ & .510 & .960 & .564 & .153 & .747 & .546 & .464 & .220 & .951 & .926 & .910 \\
ApoE3 & $\mathrm{r}$ & -.212 & -.019 & -.185 & -.027 & -.150 & .163 & .111 & .026 & -.309 & -.028 & -.070 \\
& $p$ & .110 & .885 & .165 & .841 & .265 & .222 & .409 & .847 & $\mathbf{. 0 1 9}$ & .837 & .611 \\
ApoE4 & $\mathrm{r}$ & -.119 & .314 & $\mathbf{. . 5 7 0}$ & $\mathbf{- . 6 0 9}$ & .298 & -.368 & -.413 & -.075 & -.003 & .361 & .379 \\
& $p$ & .661 & .237 & $\mathbf{. 0 2 1}$ & $\mathbf{. 0 1 2}$ & .263 & .161 & .112 & .783 & .990 & .170 & .147 \\
\hline
\end{tabular}

Table 3.Pearson correlational analyses between log 10 mean cortisol and cognitive outcomes. Values in bold represent significant or marginal values of $p$ 


\begin{tabular}{|c|c|c|c|c|c|c|c|c|c|c|c|c|}
\hline & & $\begin{array}{c}\text { RAVLT } \\
\text { Total } \\
\text { Learning }\end{array}$ & $\begin{array}{c}\text { RAVLT } \\
\text { Immediate } \\
\text { Recall }\end{array}$ & $\begin{array}{c}\text { RAVLT } \\
\text { Delayed } \\
\text { Recall }\end{array}$ & $\begin{array}{c}\text { Rivermead } \\
\text { Immediate } \\
\text { Recall }\end{array}$ & $\begin{array}{c}\text { Rivermead } \\
\text { Delayed } \\
\text { Recall }\end{array}$ & $\begin{array}{c}\text { DS- } \\
\text { Forward }\end{array}$ & $\begin{array}{c}\text { DS- } \\
\text { Backward }\end{array}$ & LNS & $\begin{array}{c}\text { TMT- } \\
\text { A }\end{array}$ & $\begin{array}{c}\text { TMT- } \\
\text { B }\end{array}$ & $\begin{array}{c}\text { Stroop } \\
\text { Interfe- } \\
\text { rence }\end{array}$ \\
\hline \multirow[t]{3}{*}{ Total } & AdjR2 & .003 & -.005 & .072 & .227 & -.013 & .364 & .163 & .285 & .085 & .193 & -.012 \\
\hline & Beta & -.125 & .085 & -.289 & -.224 & -.016 & -.015 & -.022 & -.030 & -.206 & .062 & .033 \\
\hline & $p$ & .267 & .452 & .009 & .026 & .888 & .867 & .828 & .751 & .060 & .544 & .771 \\
\hline \multirow{3}{*}{ ApoE2 } & AdjR2 & -.160 & -.153 & -.121 & -.047 & -.071 & -.111 & -.127 & .551 & -.088 & -.167 & -.124 \\
\hline & Beta & -.073 & .109 & .197 & -.320 & -.287 & .218 & .185 & -.394 & .260 & -.003 & .192 \\
\hline & $p$ & .863 & .798 & .639 & .440 & .491 & .605 & .662 & .189 & .535 & .995 & .649 \\
\hline \multirow[t]{3}{*}{ ApoE3 } & AdjR2 & .032 & -.018 & .019 & .102 & .009 & .390 & .180 & .376 & .074 & .056 & -.015 \\
\hline & Beta & -.221 & -.004 & -.190 & -.086 & -.163 & .091 & .124 & -.038 & -.302 & .015 & -.063 \\
\hline & $p$ & .098 & .974 & .156 & .505 & .229 & .394 & .314 & .723 & .024 & .909 & .653 \\
\hline \multirow{3}{*}{ ApoE4 } & AdjR2 & .161 & .034 & .548 & .605 & .261 & .640 & .280 & -.065 & .190 & .068 & .083 \\
\hline & Beta & -.032 & .314 & -.491 & -.518 & .221 & .010 & -.250 & -.075 & -.096 & .361 & .379 \\
\hline & $p$ & .895 & .237 & .015 & .008 & .343 & .956 & .303 & .783 & .690 & .170 & .147 \\
\hline
\end{tabular}

Table 4. Regression analyses with cortisol as predictor and cognitive outcomes as dependent variables. Values in bold represent significant or marginal values of $p$ 